\title{
Toward a personalized pharmacotherapy of respiratory diseases
}

\section{Paolo Montuschi*}

Department of Pharmacology, Faculty of Medicine, Catholic University of the Sacred Heart, Rome, Italy

*Correspondence: pmontuschi@rm.unicatt.it

Many respiratory diseases, but this is also true for other diseases, including asthma and chronic obstructive pulmonary disease (COPD), should be more properly defined as syndromes as they are characterized by a high degree of heterogeneity. This heterogeneity is largely responsible for the variability in drug response. A striking example is COPD where patients with chronic bronchitis or emphysema who are completely different are classified under the same nosographic entity, principally on the basis of functional characteristics. Pharmacological response in so different clinical and pathological phenotypes can be very variable even when the same drug, at the same dose, for the same duration of treatment is administered. This can make it difficult the interpretation of clinical trials in patients with COPD. Identification of patients who are more likely to respond to pharmacological treatment can increase the risk/benefit ratio in the individual patients and reduce health care costs. A promising example is the association between leukotriene receptor antagonists (LTRA) response in patients with asthma and genetic polymorphisms of the leukotriene pathway and/or receptors. A priori identification of LTRA responders among patients with asthma, that is currently based on a therapeutic trial, would enable to exploit the therapeutic effects of this class of drugs in the responders, avoiding exposure of non-responders to unjustifiable side effects and costs. More generally, a personalized pharmacotherapy of respiratory diseases would improve management of the individual patients, in terms of greater efficacy and safety, and reduce the health care costs. Personalized pharmacotherapy requires the implementation of more sophisticated diagnostic tools that should be based on a better understanding of the pathophysiology of respiratory diseases and the development of sensitive and validated non-invasive techniques. There is no doubt that chronic airway inflammation has a pivotal pathophysiological role in asthma. The most convincing evidence for that is the fact that anti-inflammatory drugs such as inhaled glucocorticoids are the most effective long-term treatment for asthma control. COPD is also characterized by lung inflammation, but the efficacy of glucocorticoids in this disease is much lesser than in asthma indicating a different type of inflammation, relatively resistant to these drugs. At present, assessment of airway inflammation is mainly based on invasive techniques including bronchoscopy and bronchial biopsies that cannot be used routinely or conventional diagnostic procedures including clinical assessment, pulmonary function testing, bronchial challenge tests, and patient reported outcomes that are indirect measures of inflammation and/or lack sensitivity. In patients with asthma, measurement of fractional exhaled nitric oxide $\left(\mathrm{F}_{\mathrm{E}} \mathrm{NO}\right)$ is a standardized, validated and well-accepted non-invasive surrogate marker of airway inflammation. $\mathrm{F}_{\mathrm{E}} \mathrm{NO}$ analyzers provide immediate results and are cleared by the US Food and Drugs Administration for assessing airway inflammation and monitoring therapy in patients with asthma. Compared with asthma control based on conventional outcomes, asthma control based on $\mathrm{F}_{\mathrm{E}} \mathrm{NO}$ measurement enables a reduction of daily doses of inhaled glucocorticoids of about $40 \%$ with similar efficacy and reduced costs and, likely, side effects. Although the clinical utility of this technique is still debated, many clinical trials on antiasthmatic pharmacological treatment include $\mathrm{F}_{\mathrm{E}} \mathrm{NO}$ among the outcome measures due to the importance of assessing drug effects based on their mechanisms of action, e.g., the effects of anti-inflammatory drugs on surrogate markers of inflammation. Analysis of eosinophil counts in induced sputum is a direct measure of airway inflammation that can be used for identifying phenotypes of patients with asthma and COPD and predicting response to inhaled glucocorticoids. However, this technique is difficult to be used routinely as it is semiinvasive, is not well-accepted to patients, requires specialized staff and facilities, and is unfeasible in children. Advances in our understanding of the clinical pharmacology of drugs for respiratory diseases should be attainable using measurements of validated biomarkers. Measurement of $\mathrm{F}_{\mathrm{E}} \mathrm{NO}$ was a major breakthrough in patients with asthma and a relevant example of a surrogate marker of airway inflammation that translated effectively from basic research, through validation and standardization of the technique, to clinical setting. However, $\mathrm{F}_{\mathrm{E}} \mathrm{NO}$ is only one surrogate marker of airway inflammation, useful in patients with asthma, particularly those with airway eosinophilia, but unlikely to reflect the whole complexity of the inflammatory process in respiratory diseases and its multiple expressions within a given disease/syndrome. To better understand the pathophysiology of respiratory diseases, and its phenotypes, a molecular approach to the individual patients based on omics technologies is being implemented. These technologies include genomics, transcriptomics, proteomics, lipidomics, metabolomics, and brethomics, a new omics technology that aims at identifying and quantifying breath biomolecules by reference analytical techniques and identifying selective profiles of breath volatile organic compounds by electronic nose, a chemical sensor array. Omics technologies, that require close interdisciplinary interactions (bioengineering, biophysics, bioinformatics), could have important implications for the pharmacological treatment of respiratory diseases: (1) identification of subphenotypes of patients with respiratory diseases that would facilitate the implementation of a tailored, personalized, pharmacotherapy; (2) unraveling novel potential targets for pharmacological intervention and development of new drugs; (3) a better understanding of the mechanism(s) of action of 
currently available drugs that would result in a more rational use of these drugs and could suggest new pharmacological strategies; (4) the evidence of ongoing pulmonary inflammation in patients with no symptoms and maintained lung function that might require starting pharmacological therapy.

Randomized clinical trials are still the best tool for assessing the pharmacological profiles of existing and new drugs, but their conclusions apply to large patient populations. The evidence of a large variability in drug response raises the issue of the best treatment for the individual patient and paves the ave- nue to a personalized pharmacotherapy. This requires a "handprint" for each single patient that should be based on the combination of conventional and novel techniques for the diagnosis and monitoring of pharmacological therapy and robust systems biology for data analysis. Systems biology is also required for an unbiased approach to discovery of new biomarkers and/or pharmacological targets, which in turn might improve characterization of patients and their treatment.

One of the major challenges in respiratory medicine is the identification of a personalized, tailored, pharmacotherapy that would optimize efficacy, tolerability, safety, and costs. This requires a focus on the individual patient, interdisciplinary work, and good, independent, research.

Received: 07 October 2010; accepted: 08 October 2010; published online: 05 November 2010.

Citation: Montuschi P (2010) Toward a personalized pharmacotherapy of respiratory diseases. Front. Pharmacol. 1:131. doi: 10.3389/fphar.2010.00131

This article was submitted to Frontiers in Pharmacotherapy of Respiratory Diseases, a specialty of Frontiers in Pharmacology.

Copyright (c) 2010 Montuschi. This is an open-access article subject to an exclusive license agreement between the authors and the Frontiers Research Foundation, which permits unrestricted use, distribution, and reproduction in any medium, provided the original authors and source are credited. 